\title{
Effects of initially adsorbed proteins on substrate surfaces during multilayer heterogeneous protein adsorption
}

Atsunori Sonoi*1, Ippei Furikado², and Kazuhiko Ishihara*3

${ }^{1}$ Personal Health Care Products Research Laboratory, Kao Corporation, 2-1-3 Bunka, Sumida-ku, Tokyo 131-8501, Japan; ${ }^{2}$ Analytical Science Research Laboratories, Kao Corporation, 1334 Minato,

Wakayama 640-8580, Japan; ${ }^{3}$ Department of Materials Engineering and Department of Bioengineering, School of Engineering, The University of Tokyo, 7-3-1 Hongo, Bunkyo-ku, Tokyo 113-8656, Japan

\section{Corresponding Authors}

Atsunori Sonoi - Phone: +81 35630 9750; Email: sonoi.atsunori@kao.com

Kazuhiko Ishihara - Phone: Tel: +81 35841 7124; Email: ishihara@mpc.t.u-tokyo.ac.jp 

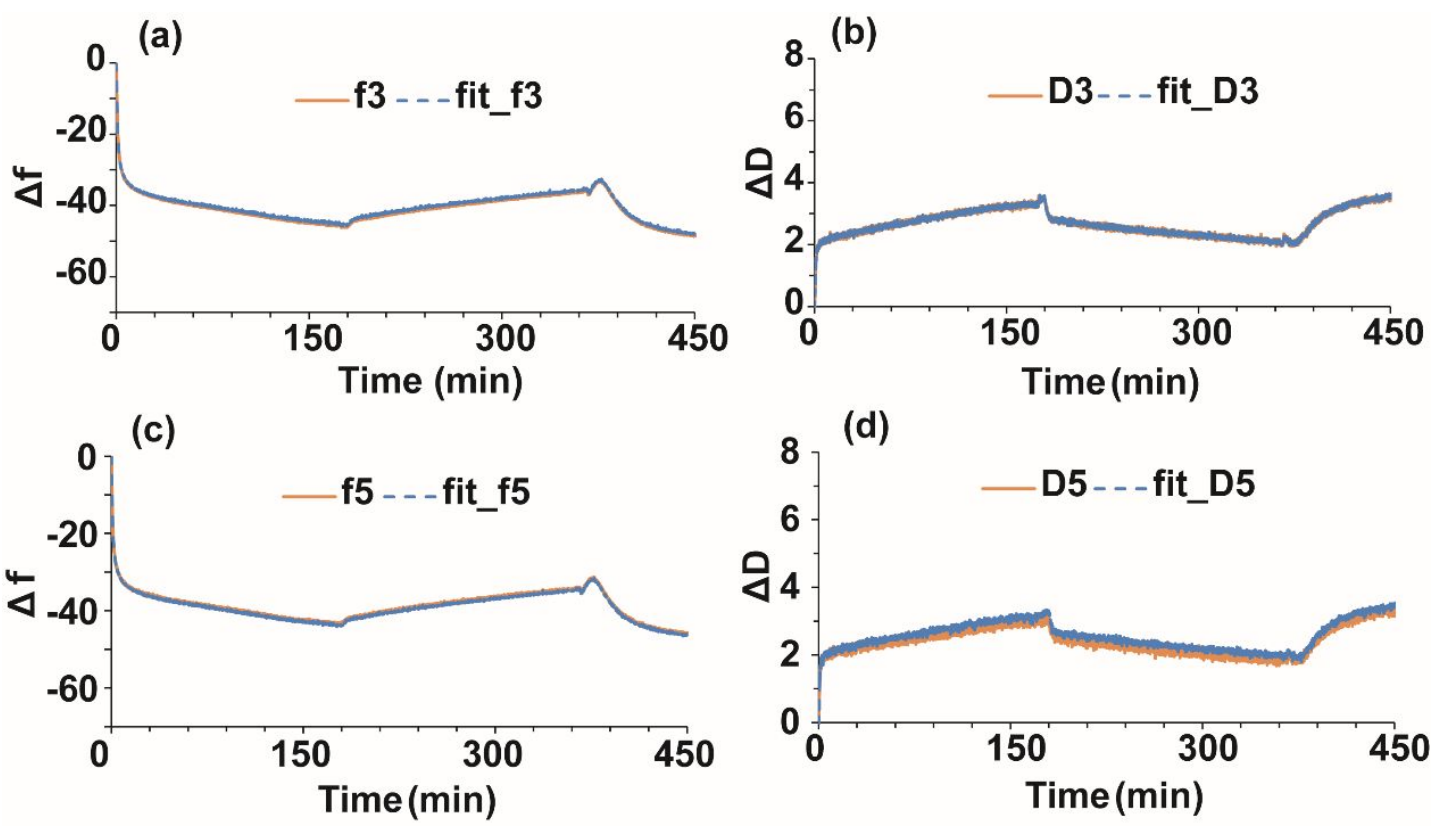

Figure S-1-1. Viscoelastic model analysis of protein adsorption of QCM-D data when BSA concentration applied was $1.7 \mathrm{mg} / \mathrm{mL}$. The $\Delta \mathrm{f}$ data of (a) third and (b) fifth overtone and the $\Delta \mathrm{D}$ of (c) third and (d) fifth overtone were used for fitting. The data which was shown each by orange line and blue dashed line is experimental data and fitted data.
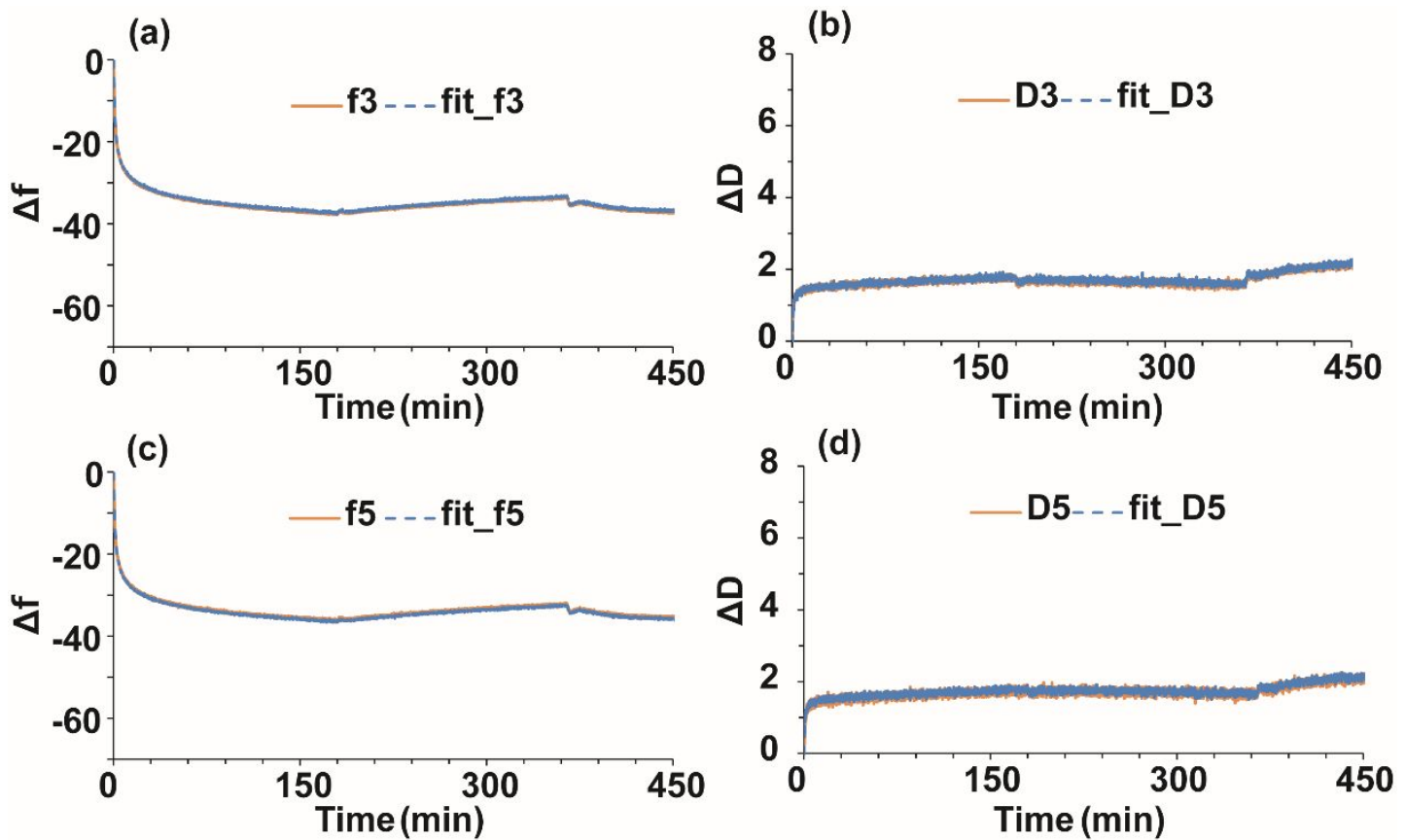

Figure S-1-2. Viscoelastic model analysis of protein adsorption of QCM-D data when BSA concentration applied was $0.43 \mathrm{mg} / \mathrm{mL}$. The $\Delta \mathrm{f}$ data of (a) third and (b) fifth overtone and the $\Delta \mathrm{D}$ of (c) third and (d) fifth overtone were used for fitting. The data which was shown each by orange line and blue dashed line is experimental data and fitted data. 
(a)
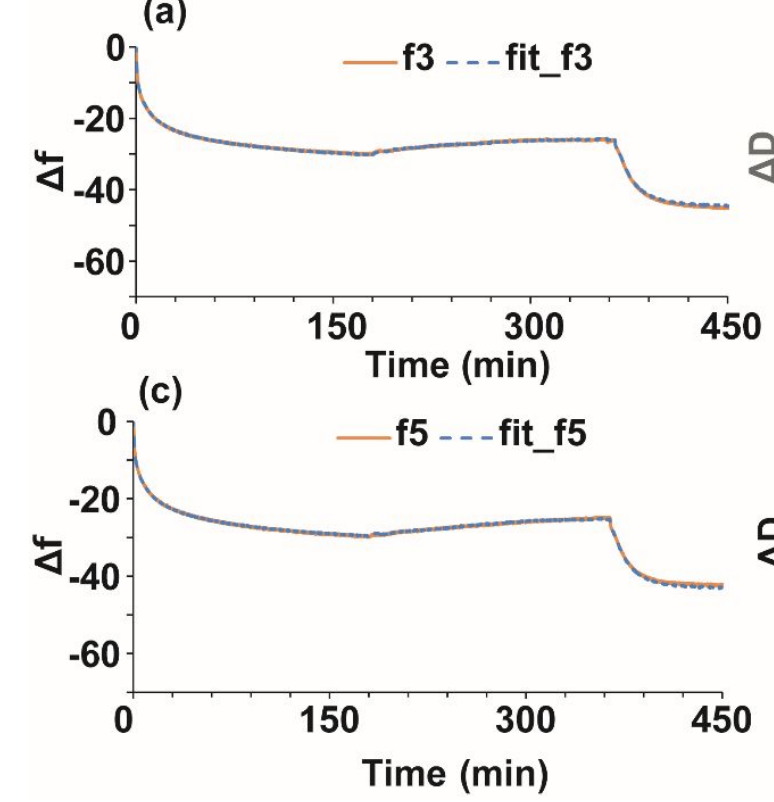

(b)

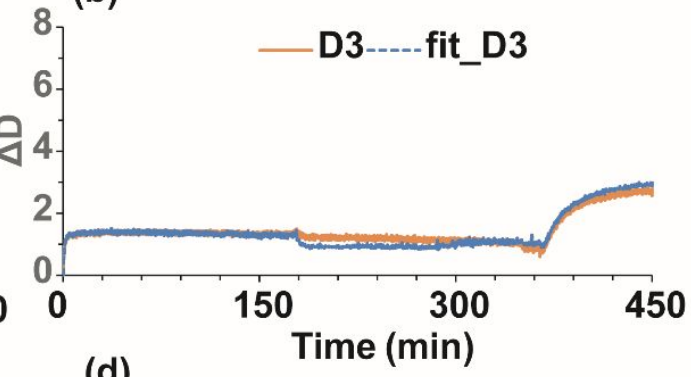

(d)

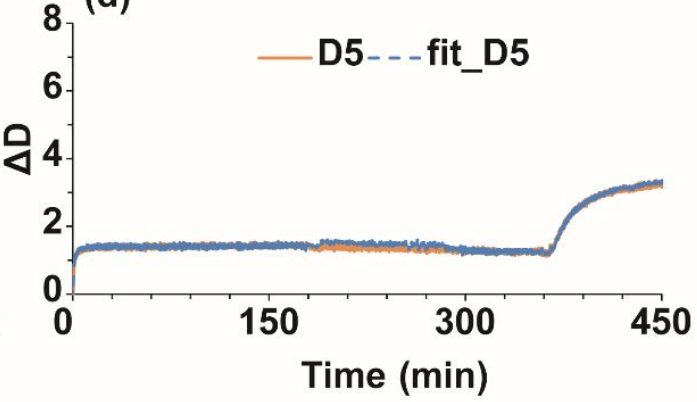

Figure S-1-3. Viscoelastic model analysis of protein adsorption of QCM-D data when BSA concentration applied was $0.22 \mathrm{mg} / \mathrm{mL}$. The $\Delta \mathrm{f}$ data of (a) third and (b) fifth overtone and the $\Delta \mathrm{D}$ of (c) third and (d) fifth overtone were used for fitting. The data which was shown each by orange line and blue dashed line is experimental data and fitted data.
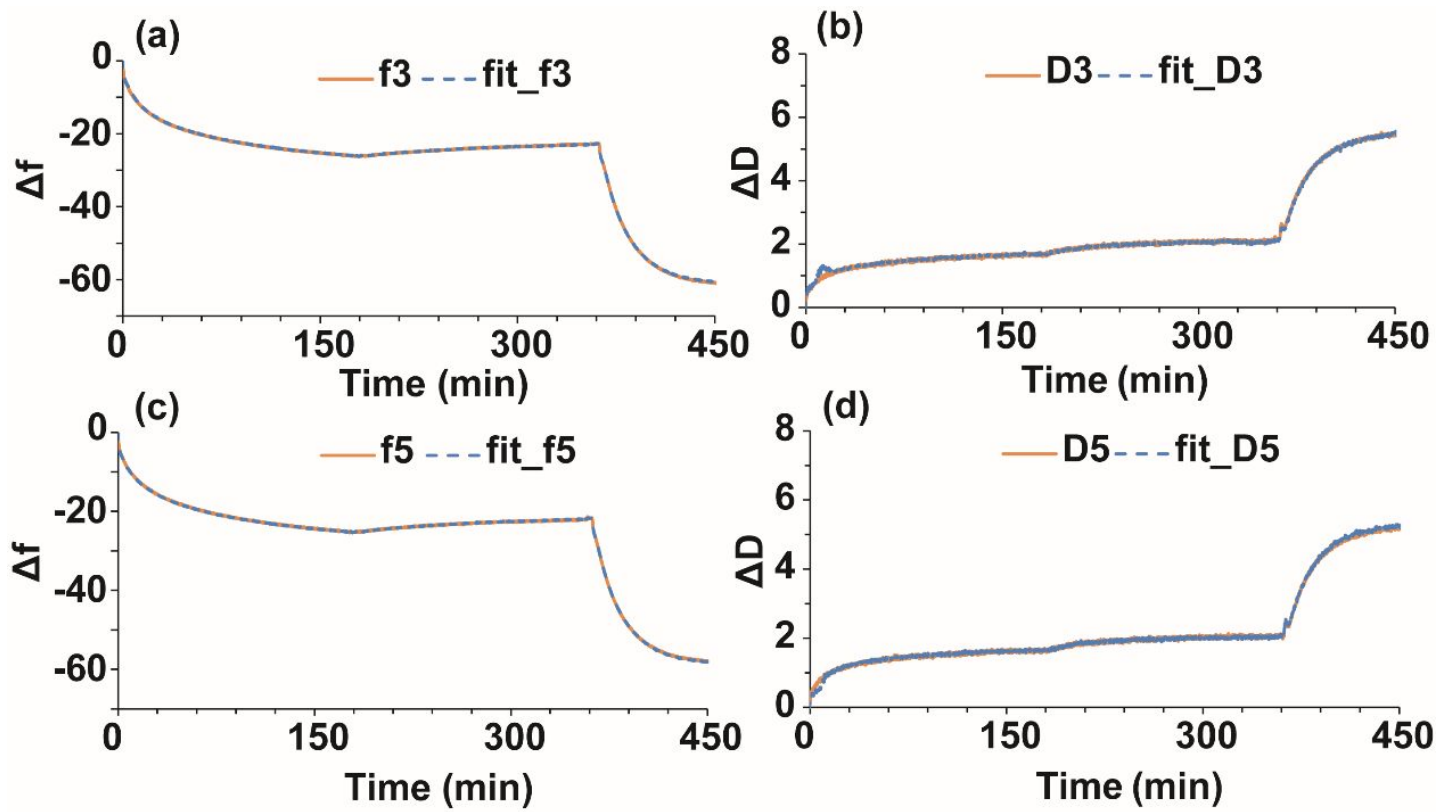

Figure S-1-4. Viscoelastic model analysis of protein adsorption of QCM-D data when BSA concentration applied was $0.086 \mathrm{mg} / \mathrm{mL}$. The $\Delta \mathrm{f}$ data of (a) third and (b) fifth overtone and the $\Delta \mathrm{D}$ of (c) third and (d) fifth overtone were used for fitting. The data which was shown each by orange line and blue dashed line is experimental data and fitted data. 

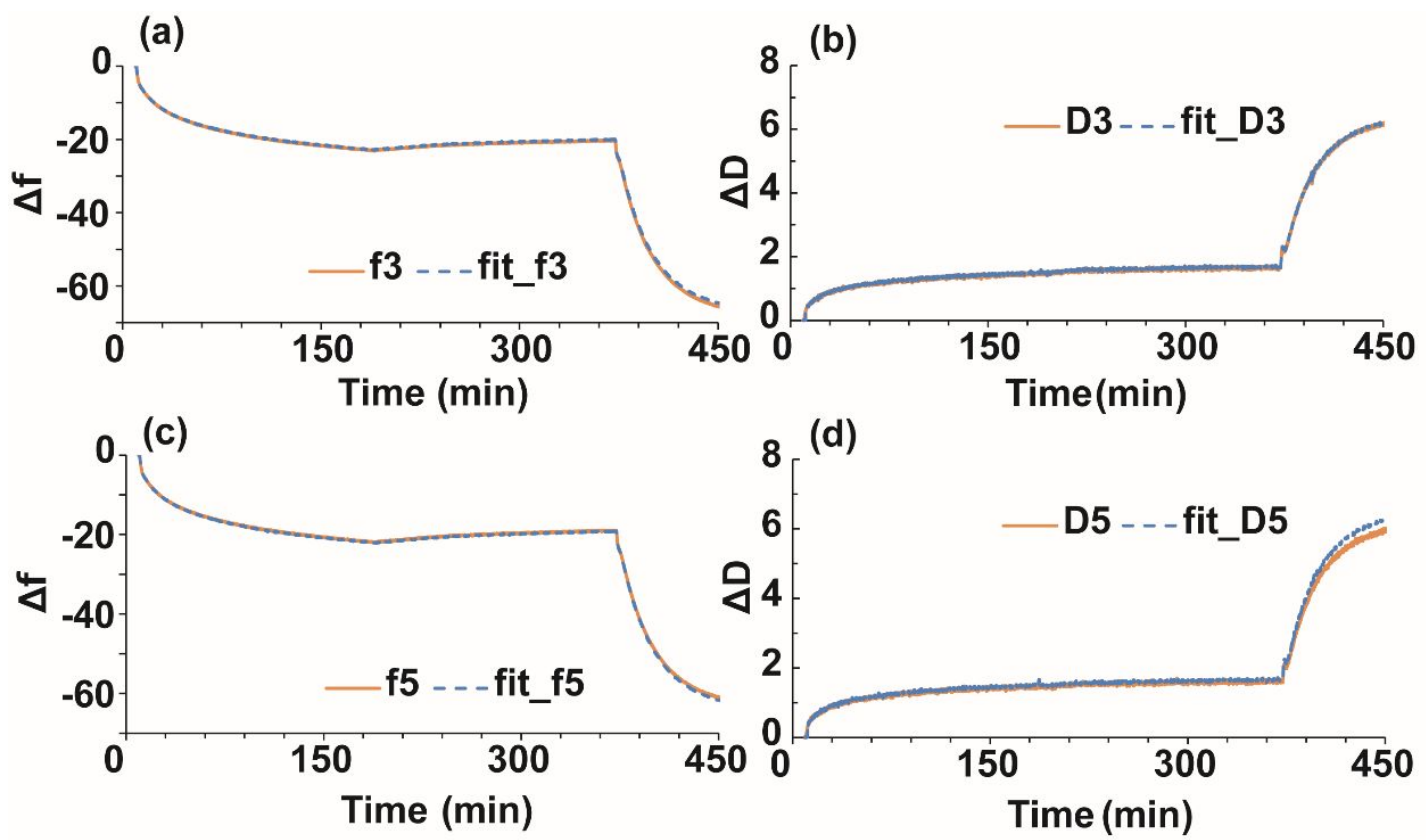

Figure S-1-5. Viscoelastic model analysis of protein adsorption of QCM-D data when BSA concentration applied was $0.043 \mathrm{mg} / \mathrm{mL}$. The $\Delta \mathrm{f}$ data of (a) third and (b) fifth overtone and the $\Delta \mathrm{D}$ of (c) third and (d) fifth overtone were used for fitting. The data which was shown each by orange line and blue dashed line is experimental data and fitted data. 


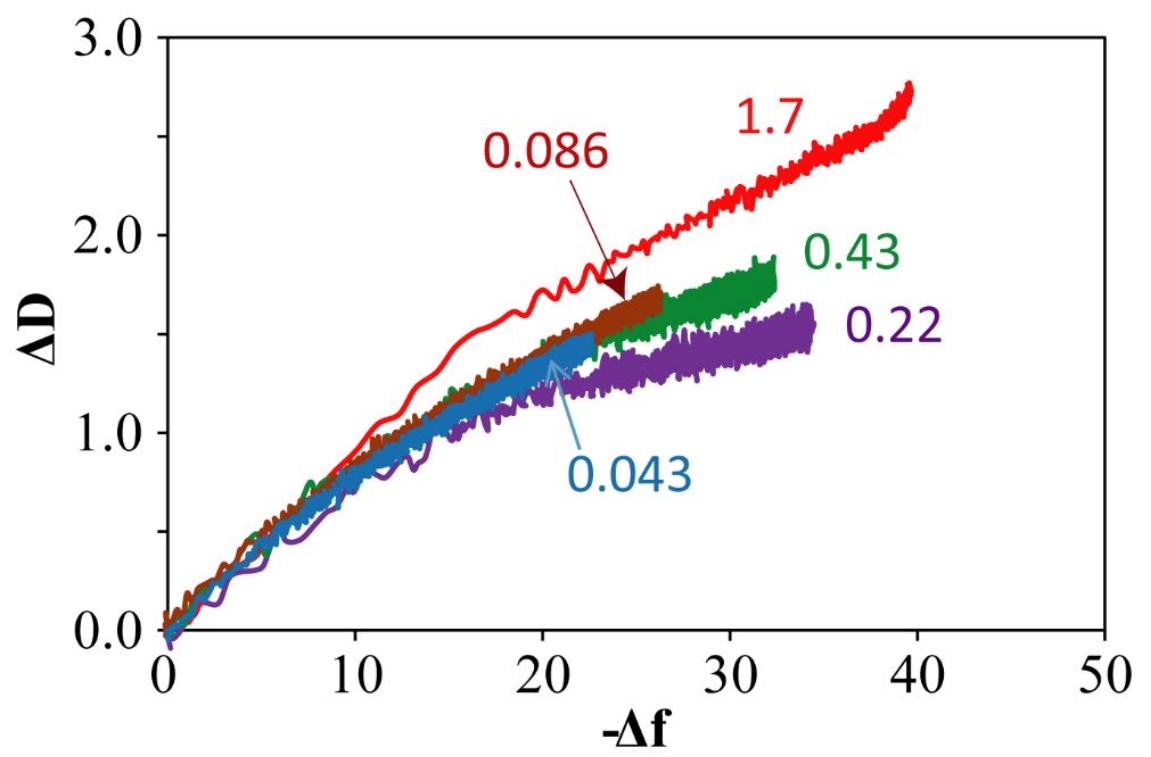

Figure S-2. $\Delta \mathrm{D} / \Delta \mathrm{f}$ plot of third overtone for the adsorption of BSA onto carboxylate SAM surfaces. The values in the graph are BSA treatment concentrations in $\mathrm{mg} / \mathrm{mL}$. 